\title{
CONTRACTION OF WALSH FOURIER SERIES
}

\section{CHINAMI WATARI}

The purpose of this note is to prove an analogue for Walsh Fourier series (abbreviated WFS) of a theorem of R. P. Boas [1]. For basic properties of Walsh functions, the reader is referred to N. J. Fine [2].

We begin with some notations and definitions:

The Walsh functions are denoted by $\psi_{n}(x)(n=0,1,2, \cdots)$ and considered in the "real version."

A complex-valued function $T(z)$ of a complex variable $z$ is called a contraction if it satisfies the inequality

$$
\left|T\left(z_{1}\right)-T\left(z_{2}\right)\right| \leqq\left|z_{1}-z_{2}\right| \text {. }
$$

An integrable function $f(x)^{1}$ is contractible if it has absolutely convergent WFS and the same is true for $T(f(x))$ where $T$ is any contraction.

Our theorem now reads as follows:

THEOREM. Let $\left\{\omega_{n}\right\}$ be a sequence of non-negative numbers with $\sum_{n=1}^{\infty} \omega_{n}<\infty$ and

$$
\sum_{n=1}^{\infty} n^{-3 / 2}\left(\sum_{\nu=1}^{n} \nu^{2} \omega_{\nu}^{2}\right)^{1 / 2}+\sum_{n=1}^{\infty} n^{-1 / 2}\left(\sum_{\nu=n}^{\infty} \omega_{\nu}^{2}\right)^{1 / 2}<\infty .
$$

Then an integrable function $f(x)$ with

$$
f(x) \sim \sum_{n=0}^{\infty} c_{n} \psi_{n}(x), \quad\left|c_{n}\right| \leqq \omega_{n}
$$

is contractible.

The proof of this theorem is essentially the same as Boas', the only difference being Lemma 3 below, in which an elementary property of Walsh function is used.

Lemma 1. For a non-negative sequence $\left\{\omega_{n}\right\},(1)$ is equivalent to

$$
\sum_{n=1}^{\infty} 2^{-n / 2}\left(\sum_{\nu=1}^{2^{n}-1} 2^{2 k} \omega_{\nu}^{2}\right)^{1 / 2}+\sum_{n=1}^{\infty} 2^{n / 2}\left(\sum_{\nu=2^{n}}^{\infty} \omega_{\nu}^{2}\right)^{1 / 2}<\infty
$$

where $k=k(\nu)$ is the integer satisfying $2^{k} \leqq \nu<2^{k+1}$.

Received by the editors December 27, 1962.

1 We consider measurable functions with period 1 only; thus the integrability is meant over an interval of length 1 , say $[0,1)$. 
Proof. The equivalence between

$$
\sum_{n=1}^{\infty} n^{-1 / 2}\left(\sum_{\nu=n}^{\infty} \omega_{\nu}^{2}\right)^{1 / 2}<\infty \text { and } \sum_{n=1}^{\infty} 2^{n / 2}\left(\sum_{\nu=2^{n}}^{\infty} \omega_{\nu}^{2}\right)^{1 / 2}<\infty
$$

is nothing but Cauchy's condensation theorem. On the other hand,

$$
\begin{aligned}
& \sum_{n=1}^{\infty} n^{-3 / 2}\left(\sum_{\nu=1}^{n} \nu^{2} \omega_{\nu}^{2}\right)^{1 / 2} \\
& =\sum_{j=0}^{\infty} \sum_{n=2^{j}}^{2^{j+1}-1} n^{-3 / 2}\left(\sum_{\nu=1}^{n} \nu_{\nu}^{2} \omega_{\nu}^{2}\right)^{1 / 2} \leqq \sum_{j=0}^{\infty} 2^{-3 j / 2} \sum_{n=2^{j}}^{2^{j+1}-1}\left(\sum_{\nu=1}^{n} \nu^{2} \omega_{\nu}^{2}\right)^{1 / 2} \\
& \leqq A \sum_{j=0}^{\infty} \sum_{n=2^{j}}^{2^{j+1}-1} 2^{-3 j / 2}\left(\sum_{\nu=1}^{2^{j+1}-1} 2^{2 k} \omega_{\nu}^{2}\right)^{1 / 2}=A \sum_{j=0}^{\infty} 2^{-j / 2}\left(\sum_{\nu=1}^{2^{j+1}-1} 2^{2 k} \omega_{\nu}^{2}\right)^{1 / 2} \\
& \leqq A \sum_{j=1}^{\infty} 2^{-j / 2}\left(\sum_{\nu=1}^{2^{j}-1} 2^{2 k} \omega_{\nu}^{2}\right)^{1 / 2},
\end{aligned}
$$

and

$$
\begin{aligned}
\sum_{j=0}^{\infty} \sum_{n=2^{j}}^{2^{j+1}-1} n^{-3 / 2}\left(\sum_{\nu=1}^{n} \nu^{2} \omega_{\nu}^{2}\right)^{1 / 2} \geqq A \sum_{j=0}^{\infty} \sum_{n=2^{j}}^{2^{j+1}-1} 2^{-3 j / 2}\left(\sum_{\nu=1}^{n} 2^{2 k} \omega_{\nu}^{2}\right)^{1 / 2} \\
\geqq A \sum_{j=0}^{\infty} 2^{-3 j / 2} \sum_{n=2^{j}}^{2^{j+1}-1}\left(\sum_{\nu=1}^{2^{j-1}} 2^{2 k} \omega_{\nu}^{2}\right)^{1 / 2} \\
=A \sum_{j=0}^{\infty} 2^{-j / 2}\left(\sum_{\nu=1}^{2^{j}-1} 2^{2 k} \omega_{\nu}^{2}\right)^{1 / 2} \text { q.e.d. }
\end{aligned}
$$

Lemma 2. For any non-negative sequence $\left\{a_{\nu}\right\}$,

$$
\sum_{\nu=1}^{\infty} a_{\nu} \leqq \sum_{n=1}^{\infty} 2^{-n / 2}\left(\sum_{\nu=1}^{2^{n}-1} 2^{2 k} a_{\nu}^{2}\right)^{1 / 2}
$$

Proof.

$$
\begin{aligned}
\sum_{\nu=1}^{\infty} a_{\nu} & =\sum_{j=0}^{\infty} \sum_{\nu=2^{j}}^{2^{j+1}-1} a_{\nu}=\sum_{j=0}^{\infty} 2^{j} \sum_{\nu=2^{j}}^{2^{j+1}-1} a_{\nu} \sum_{n=j+1}^{\infty} 2^{-n} \\
& =\sum_{n=1}^{\infty} 2^{-n} \sum_{j=0}^{n-1} 2^{j} \sum_{\nu=2^{j+1}}^{2^{j+1}} a_{\nu}=\sum_{n=1}^{\infty} 2^{-n} \sum_{j=0}^{n-1} \sum_{\nu=2^{j}}^{2^{j+1}-1} 2^{k} a_{\nu} \\
& =\sum_{n=1}^{\infty} 2^{-n} \sum_{\nu=1}^{2 n-1} 2^{k} a_{\nu} \leqq \sum_{n=1}^{\infty} 2^{-n} \cdot 2^{n / 2}\left(\sum_{\nu=1}^{2^{n}-1} 2^{2 k} a_{\nu}^{2}\right)^{1 / 2} \\
& =\sum_{n=1}^{\infty} 2^{-n / 2}\left(\sum_{\nu=1}^{2 n-1} 2^{2 k} a_{\nu}^{2}\right)^{1 / 2}, \quad \text { q.e.d }
\end{aligned}
$$


LEMMA 3. Let $f(x)=\sum_{\nu=0}^{\infty} c_{\nu} \psi_{\nu}(x)$ with $\sum_{\nu=0}^{\infty}\left|c_{\nu}\right|^{2}<\infty$ and let $g(x)$ $\sim \sum_{\nu=0}^{\infty} a_{\nu} \psi_{\nu}(x)$ be a contraction of $f(x)$. Then

$$
\sum_{\nu=1}^{2^{n}-1} 2^{2 k}\left|a_{\nu}\right|^{2} \leqq A \sum_{\nu=1}^{2^{n}-1} 2^{2 k}\left|c_{\nu}\right|^{2}+A 2^{n} \sum_{\nu=2^{n}}^{\infty}\left|c_{\nu}\right|^{2} .
$$

Proof. For any $h, 0<h<1$, choose the integer $m$ so that

$$
2^{-m-1} \leqq h<2^{-m} \text {. }
$$

We have, by the definition of Walsh functions,

$$
\psi_{\nu}(h)=-1 \quad\left(2^{m} \leqq \nu<2^{m+1}\right) .
$$

From Parseval's relation combined with (2), it follows that

$$
\begin{aligned}
4 \sum_{\nu=2^{m}}^{2^{m+1}-1}\left|a_{\nu}\right|^{2} & \leqq \sum_{\nu=2^{m}}^{\infty}\left|a_{\nu}\right|^{2}\left|1-\psi_{\nu}(h)\right|^{2}=\int_{0}^{1}|g(x+h)-g(x)|^{2} d x \\
& \leqq \int_{0}^{1}|f(x+h)-f(x)|^{2} d x=\sum_{\nu=2^{m}}^{\infty}\left|c_{\nu}\right|^{2}\left|1-\psi_{\nu}(h)\right|^{2} \\
& \leqq 4 \sum_{\nu=2^{m}}^{\infty}\left|c_{\nu}\right|^{2} .
\end{aligned}
$$

Thus we have

$$
\sum_{\nu=2^{m}}^{2^{m+1}-1}\left|a_{\nu}\right|^{2} \leqq \sum_{\nu=2^{m}}^{\infty}\left|c_{\nu}\right|^{2}=\sum_{j=m}^{\infty} \sum_{\nu=2^{j}}^{2^{j+1}-1}\left|c_{\nu}\right|^{2} .
$$

Since (3) is true for each $m$, multiplication by $2^{2 m}$ and summation with respect to $m$ give

$$
\begin{aligned}
\sum_{m=0}^{n-1} 2^{2 m} \sum_{\nu=2^{m}}^{2^{m+1}-1}\left|a_{\nu}\right|^{2} & =\sum_{m=0}^{n-1} \sum_{\nu=2^{m}}^{2^{m+1}-1} 2^{2 k}\left|a_{\nu}\right|^{2} \leqq \sum_{m=0}^{n-1} 2^{2 m} \sum_{j=m}^{\infty} \sum_{\nu=2^{j}}^{2^{j+1}-1}\left|c_{\nu}\right|^{2} \\
& =\sum_{m=0}^{n-1} 2^{2 m} \sum_{j=m}^{n-1} \sum_{\nu=2^{j}}^{2^{j+1}-1}\left|c_{\nu}\right|^{2}+\sum_{m=0}^{n-1} 2^{2 m} \sum_{j=n}^{\infty} \sum_{\nu=2^{j}}^{2^{j+1}-1}\left|c_{\nu}\right|^{2} \\
& =\sum_{j=0}^{n-1} \sum_{\nu=2^{j}}^{2^{j+1}-1}\left|c_{\nu}\right|^{2} \sum_{m=0}^{j} 2^{2 m}+\sum_{j=n}^{\infty} \sum_{\nu=2^{j}}^{2^{j+1}-1}\left|c_{\nu}\right|^{2} \sum_{m=0}^{n-1} 2^{2 m} \\
& \leqq A \sum_{j=0}^{n-1} 2^{2 j} \sum_{\nu=2^{j}}^{2^{j+1}-1}\left|c_{\nu}\right|^{2}+A \cdot 2^{2 n} \sum_{j=n}^{\infty} \sum_{\nu=2^{j}}^{2^{j+1}-1}\left|c_{\nu}\right|^{2} \\
& \leqq A \sum_{\nu=1}^{2^{n}-1} 2^{2 k}\left|c_{\nu}\right|^{2}+A \cdot 2^{2 n} \sum_{\nu=2^{n}}^{\infty}\left|c_{\nu}\right|^{2} \text {, q.e.d. }
\end{aligned}
$$


Proof of Theorem. By Lemmas 2 and 3, we have

$$
\begin{aligned}
\sum_{n=1}^{\infty}\left|a_{\nu}\right| & \leqq A \sum_{n=1}^{\infty} 2^{-n / 2}\left(\sum_{\nu=1}^{2^{n}-1} 2^{2 k}\left|a_{\nu}\right|^{2}\right)^{1 / 2} \\
& \leqq A \sum_{n=1}^{\infty} 2^{-n / 2}\left(\sum_{\nu=1}^{2^{n}-1} 2^{2 k}\left|c_{\nu}\right|^{2}+2^{2 n} \sum_{\nu=2^{n}}^{\infty}\left|c_{\nu}\right|^{2}\right)^{1 / 2} \\
& \leqq A \sum_{n=1}^{\infty} 2^{-n / 2}\left(\sum_{\nu=1}^{2^{n}-1} 2^{2 k} \omega_{\nu}^{2}\right)^{1 / 2}+A \sum_{n=1}^{\infty} 2^{n / 2}\left(\sum_{\nu=2^{n}}^{\infty} \omega_{\nu}^{2}\right)^{1 / 2},
\end{aligned}
$$

which is convergent by Lemma 1 and the assumption of Theorem.

\section{REFERENCES}

1. R. P. Boas, Beurling's test for absolute convergence of Fourier series, Bull. Amer. Math. Soc. 66 (1960), 24-27.

2. N. J. Fine, On Walsh functions, Trans. Amer. Math. Soc. 65 (1949), 372-414.

TôHoku University, Sendai, Japan 